Horizons philosophiques

\title{
Christoph Menke : herméneutique, déconstruction et raison dans le problème de l'expérience esthétique
}

\section{Michel Ratté}

Volume 7, numéro 2, printemps 1997

L’héritage de l'herméneutique

URI : https://id.erudit.org/iderudit/801047ar

DOI : https://doi.org/10.7202/801047ar

Aller au sommaire du numéro

Éditeur(s)

Collège Édouard-Montpetit

ISSN

1181-9227 (imprimé)

1920-2954 (numérique)

Découvrir la revue

Citer cet article

Ratté, M. (1997). Christoph Menke : herméneutique, déconstruction et raison dans le problème de l'expérience esthétique. Horizons philosophiques, 7(2),

107-126. https://doi.org/10.7202/801047ar d'utilisation que vous pouvez consulter en ligne. 


\section{CHRISTOPH MENKE : \\ HERMÉNEUTIQUE, \\ DÉCONSTRUCTION \\ ET RAISON DANS LE PROBLÈME \\ DE L'EXPÉRIENCE ESTHÉTIQUE}

Dans son important livre La souveraineté de l'art. L'expérience esthétique après Adorno et Derrida', Christoph Menke réussit à faire bouger dans un sens inattendu la lourde constellation épistémologique constituée par l'herméneutique, la déconstruction et le problème de la raison. II consacre notamment plusieurs pages à la discussion des identités et différences du concept de compréhension véhiculé d'une part dans l'herméneutique philosophique et d'autre part dans l'art moderne. Cette discussion part de son idée centrale d' «esthétique de la négativité" de la dialectique négative d'Adorno qui représente le fond séminal de l'interprétation adornienne de l'art'2 et qui est ici traduite dans les termes de la subversion sémiotique de la déconstruction derridienne.

Après avoir succinctement présenté le contexte théorique où s'insère la discussion sur l'herméneutique(I), je dégagerai les lignes de forces de ce qu'il appelle la «controverse entre l'esthétique de la négativité et l'herméneutique». Menke veut montrer que les bases de l'ontologie de la compréhension ne sont pas en mesure de rendre justice au mode d'investissement de la compréhension esthétique que la modernité esthétique fait émerger (II). Pour terminer, je voudrais poser la question de savoir si la théorie de Menke ne doit pas trouver un fondement ailleurs que dans l'idée d'une souveraineté de l'art auto-fondé, même en tant que déconstruction retrouvant ses limites et évitant les conséquences anti-rationalistes du derridianisme(III).

1. Christoph Menke, La souveraineté de l'art. L'expérience esthétique après Adorno et Derrida, trad. Pierre Rusch, Paris, Armand Colin, coll. Théories, 1993. Pour ne pas alourdir la lecture, les références subséquentes à ce titre apparaîtront dans le corps du texte entre parenthèses mentionnant le nom de l'auteur et la ou les page(s) citée(s).

2. Theodor W. Adorno, Dialectique négative, Paris : Payot, 1992; “Théorie esthétique", Paris Klincksieck, 1989. 


\section{I- La souveraineté de l'art : contexte et finalité de l'entreprise théorique de Menke}

L'intervention de Menke se situe à un carrefour théorique complexe où se rencontrent 1) le débat post-adornien sur la question de l'art dans la reprise habermassienne du projet de la théorie critique à partir de la pragmatique-communicationnelle ${ }^{3}$; 2) la critique de la conservation d'un héritage poststructuraliste comme critique radicale de la raison, qui a été sacrifié par Habermas 4 ; 3) la reprise de la critique de l'herméneutique philosophique à partir d'autres arguments que ceux d'Habermas contre Gadamer5.

Par sa reconstruction sémiotique de la théorie esthétique d'Adorno, Menke entend faire échec à la retraduction communicationnelle de cette dernière qu'a proposée A. Wellmer et qu'a entérinée Habermas. Rappelons que l'entreprise wellmérienne est rendue possible par la thèse fondamentale de Habermas selon laquelle le contenu utopique de la théorie adornienne de l'art - les traces en son sein d'une rationalité mimétique nous rappelant l'échec historique de la raison - peut être débarrassé de sa prétention critique socio-historique et compris comme la postulation contrafactuelle d'une communication sans contrainte impliquée dans tous nos actes de langage. Wellmer affirme à partir de cette thèse que l'unité de l'expérience esthétique décrite par Adorno peut être conservée dans une traduction pragmatique où interfèrent les différentes dimensions de la rationalité. Cela implique aussi que tout le contenu cognitif de l'art est rétribué au quotidien comme une chance pour sa trans-

3. Voir notamment, Jürgen Habermas, "La modernité : Un projet inachevé", dans Critique, $n^{\circ} 413$, oct. 1981; Théorie de l'agir communicationnel, tome I, Paris, Fayard, 1987, particulièrement p.31-59; "Questions et contre-questions", dans Critique, $n^{\circ}$ 493-494, 1988; Albrecht Wellmer, "Vérité- apparence-réconciliation. Adorno et le sauvetage esthétique de la modernité", in Rainer Rochlitz (dir.), Théories esthétiques après Adomo, Arles, Actes sud, 1990, pp.249-293; Martin Seel, L'art de diviser. Le concept de rationalité esthétique, Paris : Armand Colin, 1993; Rainer Rochlitz, "De l'expression au sens. Perspectives esthétiques chez Habermas", dans Revue intemationale de philosophie, $n^{\circ} 4$, décembre 1995, p.409-435;

4. Voir notamment, Jürgen Habermas, Le discours philosophique de la modernité, Paris, Gallimard, 1988, p.191-248

5. Voir la section II de cet article 
figuration : c'est dans le quotidien en tant qu'il comprend que se réalise le contenu cognitif de l'art ${ }^{6}$ - alors que chez Adorno ce contenu cognitif était essentiellement philosophique.

Bien que Menke soit d'accord avec Wellmer sur le fait que c'est là la possibilité de faire éclater les «automatismes» des discours non esthétiques (Menke, p.197), il considère que Wellmer sacrifie l'autonomie propre de la compréhension inhérente à l'expérience esthétique moderne où le procès mimétique n'a pas pour finalité une certaine "plénitude» du sens comme le conçoit l'herméneutique philosophique. Wellmer ne reconnaît pas le fait que l'art comporte un contenu cognitif propre dont la souveraineté consiste précisément à être indépendant de son accomplissement dans la vie quotidienne, voire indépendant de sa simple incidence sur celle-ci.

L'autonomie de l'expérience esthétique moderne résiderait précisément dans le fait qu'elle produit un savoir déconstructiviste : elle montre entre autres le leurre qui préside aux consensus concernant les œuvres d'art car celles-ci s'offrent à la compréhension, d'une manière inhérente, comme un problème pour leur compréhension effective. Celle-ci est en fait constamment ajournée parce que chaque œuvre d'art se présente comme une scission de la lettre et du sens. Pour Menke, "percevoir des textes sur le mode esthétique cela signifie rester fidèle à leur lettre [...] la lettre n'est plus absorbée par l'esprit, elle est conservée contre l'esprit» (Menke, p.33). La lecture derridienne d'Adorno que propose Menke prétend que c'est seulement dans le renvoi de la lettre à l'esprit (et réciproquement) que l'esprit esthétique est esthétique chez Adorno. II n'y aurait pas de "vaste médiation" comme dans l'herméneutique posthégélienne mais un mouvement qui se dépasse lui-même (Menke, p.38).

Menke insiste cependant, contre Derrida, sur le fait que cette expérience de déconstruction ne peut se produire que dans la sphère esthétique. C'est que Derrida n'hésite pas à imputer le statut de signe esthétique à tous les discours

6. A. Wellmer, op. cit., p.272. 
textuels, en ne pensant toute différence entre les discours qu'analogiquement à des différences entre genres littéraires "d'un seul et même texte général» (Menke, p.194). Si Menke est d'accord avec Habermas pour critiquer cette généralisation, pour sa part, il ajoute que «dans l'expérience esthétique, la négativité nie certes tous les autres discours, mais reste totalement non pertinente pour ceux-ci, parce que la validité est limitée à l'accomplissement actuel de l'expérience esthétique" (Menke, p.191) «dans laquelle nous considérons des objets du point de vue de la beauté»(Menke, p.195).

C'est en fait sur la profondeur historique du concept philosophique de beauté dans la modernité que Menke fait reposer la question de la différenciation et de l'autonomie de l'expérience esthétique : "L'expérience contraignante de la négativité esthétique est en même temps celle du beau»(Menke, p.189) ${ }^{7}$. L'auteur crée leur lien immanent sur la base de l'idée - kantienne, selon toute vraisemblance - de l'indétermination conceptuelle du beau : «le beau est un autodépassement de l'objet, sans que ce dépassement puisse être capitalisé dans une signification arrêtée [...] le beau explicité comme image est la forme affirmative de l'hésitation prolongée entre la chose et le signe"(Menke, p.182).

L'esthétique reste cependant le fondement d'une critique de la raison. On sait que cette critique ne se fait pas sur le mode d'une intervention dans les actualisations des autres formes de discours - comme le fait la déconstruction derridienne. Mais cela n'empêcherait pas que les contextes pratiques des autres discours pourraient être transfigurés - «acquérir une nouvelle image" d'eux-mêmes comme le dit Menke - grâce à l'effectuation "souveraine» de l'expérience esthétique (Menke, p. 197) . C'est ce qu'exprime Menke en écrivant : «Par l'accomplissement souverain de l'expérience esthétique, la conception préesthétique des discours non esthétiques cède la place à une conception postesthétique. Celle-ci s'oppose certes à la première, mais elle n'est pas impliquée dans l'expérience esthétique : elle en est la conséquence" (ibidem).

7. C'est moi qui souligne 
La souveraineté de la négativité esthétique, comprise comme la possibilité d'enrichir a posteriori d'autres modes de discours devient le mobile pour faire une distinction critique entre "esthétique romantique" et "esthétique moderne". Menke entérine d'abord l'argument habermassien sur la question du rôle de l'art dans la philosophie, de Nietzsche à Adorno : on a surévalué les possibilités de l'art, en tant qu'audelà de la raison, de trouver une solution au désenchantement de la raison que produit la différenciation des rationalités. Menke prétend cependant qu'Habermas jette le bébé avec l'eau du bain. S'il était capable de faire la distinction entre le fait d'une critique de la raison (esthétique moderne) et celui de trouver une solution à l'aporie de la raison à partir d'un au-delà de la raison (esthétique romantique), Habermas serait en mesure de reconnaître que l'esthétique moderne confronte la raison moderne à un problème insoluble en le produisant elle-même (Menke, p. 285) - indépendamment de sa prétention à résoudre effectivement le problème. L'auteur conteste donc la compréhension habermassienne d'Adorno qui le range du côté des romantiques : selon Menke, Adorno aurait une lecture esthétique des autres sphères de rationalité ne jettant sur le non esthétique qu'un éclairage postesthétique. L'expérience esthétique devient pour le non esthétique «le catalyseur dans l'émergence de problèmes qui, sans l'expérience esthétique, ne pourraient ni apparaître ni être pensés» (Menke, p.286). II s'agit là d'un projet irréductiblement concurrent de celui d'Habermas. Celui-ci cherche à faire une critique de la "colonisation du monde vécu" par les processus autonomisés de rationalisation sur la base de l'appauvrissement du monde vécu que représente en soi la séparation de la raison en sous-systèmes. Ici, il incombe à l'art de produire les expériences qui auront valeur exemplaire en étant orientées vers une harmonie postmétaphysique des sphères de rationalités différenciées ${ }^{8}$. Selon Menke, la souveraineté de l'expérience esthétique $a$, chez Adorno, une fonction heuristique et tonifiante pour la critique de

8. Cf. notamment, Jürgen Habermas, Morale et communication : Conscience morale et activité communicationnelle, trad., Paris : Cerf, 1986, p.39. 
la raison comprise maintenant comme critique des "automatismes" des discours non esthétiques ${ }^{9}$.

On peut se demander si cette définition structurelle ou formelle de l'autonomie de l'expérience esthétique moderne par rapport à la question de la différenciation des rationalités n'a pas le souffle un peu court si on se met à la considérer d'un point de vue socio-historique. Où en est la possibilité pour l'institution artistique de produire aujourd'hui des critiques inattendues d'autres discours et assumées par ceux-ci10? Où est, selon l'expression de Menke, le pouvoir "contraignant» de l'expérience esthétique? Ces questions me semblent avoir un impact sur l'immanence même de cette théorie. J'y reviendrai.

\section{II- La critique menkienne de l'herméneutique}

Depuis le débat entre Gadamer et Habermas, une tâche fondamentale de la Théorie critique aura été de se comprendre elle-même comme une herméneutique critique. Rappelons que la critique de Habermas ne porte pas sur le principe de l'herméneutique selon lequel l'interprète et son objet font partie d'un même contexte historique. Habermas assume l'idée que le "préjugé» de la tradition socio-historique, sans qu'il ait un quelconque primat, conditionne l'interprétation, la compréhension. Mais il interroge l'entreprise de Gadamer voulant réhabiliter, au nom d'une ontologie de la compréhension, l'autorité de la tradition contre le discrédit qu'elle aurait subi dans l'Aufklärung ${ }^{11}$. Gadamer soutient qu'il y a des "préjugés légitimes», comme "principes de connaissance» où la séparation formelle entre

9. Chez Menke, la critique de Habermas est au fond la même que chez Martin Seel (op. cit.). L'interférence dans la raison que rend possible la "souveraineté" de l'art chez Menke est transposée dans l'immanence même du concept de "rationalité esthétique" chez Seel, concept qui embrasse l'interférence de différentes modalités de rationalités intervenant dans la discussion esthétique. Cf. ma recension du livre de Martin Seel dans Horizons Philosophiques, vol. 6, n², printemps 1996, p.149-152.

10. Cf. à cet effet aussi Rainer Rochlitz, "Sens et fonction de la subversion esthétique», Critique, n511 (1991).

11. H.-G. Gadamer, Vérité et Méthode, trad., Étienne Sacre, Seuil, 1976 (trad. partielle) p.109. Depuis la rédaction de cet article, une nouvelle traduction (intégrale) de Vérité et méthode est parue. Je me suis permis de maintenir les références à l'ancienne traduction qui est l'édition de référence du traducteur du livre de Menke. 
autorité et vérité disparaît ${ }^{12}$. Habermas conteste que l'autorité normative de la tradition soit aussi de facto source de légitimité $^{13}$. II ne remet pas en cause la facticité de la force du préjugé mais plutôt la reconnaissance du droit du préjugé, au nom de cette facticité, qui semble être devenu chez Gadamer, d'après Habermas, l'essence de l'autorité14. À la théorie de Gadamer qui mène à l'idée que la véritable autorité des traditions, celle qui est légitime en tant que source de connaissance, ne s'affirme jamais autoritairement, Habermas oppose, à partir de son anthropologie matérialiste, que

la méta-institution du langage en tant que tradition est [...] manifestement dépendante de processus sociaux qui ne viennent pas éclore au sein des contextes normatifs. Le langage est aussi un médium de la domination et du pouvoir social [...] Tant que les légitimations ne disent pas le rapport de violence dont elles rendent possible l'institutionnalisation, tant que ce rapport de violence ne fait que s'exprimer dans les légitimations, le langage est aussi idéologique. Par là il ne s'agit pas des illusions dans un langage, mais d'illusion avec le langage comme tel. L'expérience herméneutique qui se heurte à une telle dépendance du contexte symbolique par rapport à des relations de fait se change en critique de l'idéologie ${ }^{15}$.

Cela dit, Habermas aura beaucoup de difficultés à traduire et à spécifier la tâche de la Théorie critique, à titre d'herméneutique critique, en regard de la sphère autonomisée de l'expérience esthétique - ce qui le mènera à suivre la piste wellmérienne d'une esthétique de la réception construite dans la retraduction pragmatique (évoquée plus tôt) de la théorie de l'art d'Adorno ${ }^{16}$.

12. Ibid., p.115 et sq.

13. Jürgen Habermas, Logique des sciences sociales et autres essais, trad., Rainer Rochlitz, Paris : P.U.F., 1987, p.212-215.

14. Jean-Marc Ferry, Habermas. L'éthique de la communication, Paris : P.U.F.,1987, p.131.

15. Jürgen Habermas, Zu Gadamers "Wahrheit und Methode", in Hermeneutik und Ideologiekritik, Frankfort, Suhrkamp, 1971, p.49, cit. et trad. par J.-M. Ferry, opus cit., p. 132.

16. J'ai aussi abordé ce problème dans "Autonomie de l'art et communication. Le problème de l'œuvre d'art dans la théorie socio-communicationnelle postadornienne" dans Société, $n^{\circ} 15 / 16$, été 1996, p.329-390 (plus précisément dans les pp.335-359). 
Pour sa part, l'intervention de Menke vient remettre en question la possibilité même de poser le problème de l'esthétique post-adornienne dans les termes de l'herméneutique critique en mettant en doute la pertinence même du concept d'herméneutique pour la compréhension de l'expérience esthétique. Non seulement Menke entérine-t-il la critique de Bubner' visant à montrer l'hétéronomie de l'argument fondamental de l'ontologie de la compréhension qui donne une fonction exemplaire à l'expérience esthétique ${ }^{17}$, mais il entend aller plus loin en critiquant la surcharge sémantique que cette ontologie impose à l'expérience esthétique dans sa décision fondamentale de l'envisager comme acte de compréhension achevé (Menke, p.123).

a) Considérations préliminaires sur l'autosubversion de la formation des signifiants et la critique de l'idée de polysémie

L'ajournement du procès de compréhension propre à l'expérience esthétique implique qu'il y ait une structure spécifique au symbole qui soit l'occasion de cette expérience. On a déjà dit que Menke avait défini l'œuvre d'art en terme déconstructiviste comme un symbole qui fait jouer «la lettre contre l'esprit». Menke souligne cependant que la «lettre» n'est pas la simple littéralité (ou le "signifiant" au sens de la linguistique saussurienne), mais, selon le mot de Valéry à propos du poème, "l'hésitation prolongée entre le sens et le son". Pour Menke, la «lettre» est tendue vers le sens et sa simple factualité matérielle (Menke, p.51).

Menke montre également ce que l'on pourrait appeler le non-lieu du débat entre N. Goodman et G. Patzig ${ }^{18}$, c'est-à-dire le débat sur le statut du symbole esthétique dans les théories générales des symboles où la matérialité des symboles esthétiques est tout de même thématisée. Dans une critique croisée des deux auteurs, Menke veut démontrer que la distinction

17. Rüdiger Bubner, "De quelques conditions devant être remplies par une esthétique contemporaine" dans Théories esthétiques après Adorno, op. cit. pp.79128.

18. Nelson Goodman, Langages de l'art. Approche d'une théorie des symboles, Ed. J. Chambon, 1990; Günther Patzig, "Über den Ontologischen Status von Kunstwerken", in F.W. Korff (éd.), Redlisches Denken, Stuttgart-Bad Cannstatt, 1981, p.114 et sq. 
goodmanienne entre les arts allographiques (qui présupposent un code conventionnel permettant la reproduction des œuvres) et autographiques (qui n'en présupposent pas - comme la peinture) est maintenable contre la critique de Patzig qui ne veut pas de cette distinction (bien que, par ailleurs, Menke pense que ce dernier critique légitimement l'idée goodmanienne selon laquelle l'œuvre autographique est réductible à un "objet concret»). Cela dit, Menke souligne que le fait que des automatismes conventionnels interviennent dans les arts allographiques ne signifie pas qu'ils en déterminent les signifiants esthétiques. Pour Menke, la distinction goodmanienne n'est pas déterminante parce qu'elle ne renvoie qu'à la manière dont une œuvre se manifeste alors que ce qu'il vise avec son idée d'hésitation entre le sens et le matériau se rapporte au choix que nous faisons dans l'acte de compréhension esthétique (Menke, p.62). «L'hésitation du signifiant entre le sens et le matériau est un effet de l'ajournement perpétuel de la formation des signifiants dans l'expérience esthétique; seul ce processus permet de comprendre que l'objet esthétique se constitue dans l'hésitation entre les deux pôles»(Menke, p.64). "S'engager dans cette expérience c'est donc envisager la formation des signifiants dans une perspective nouvelle qui la ruine. Car si le matériau lui-même ne fournit aucun critère quant aux sélections dont il doit faire l'objet, et si les signes esthétiques ne s'inscrivent dans aucun contexte préalable, alors la reprise processuelle de la formation des signifiants est nécessairement vouée à l' échec" (Menke, p.71).

Menke montre également que la théorie de cette processualité négative immanente à l'expérience esthétique ne peut pas se confondre avec une théorie de la polysémie (Menke, p.79 et sq). Alors que les théories de la polysémie constatent la polysémie de l'extérieur, l'esthétique de la négativité montre l'auto-subversion du sens immanent à l'expérience esthétique. Les objets esthétiques n'acquièrent leur prétendue plurivocité que de l'extérieur, comme par exemple, les événements deviennent plurivoques sous le regard rétrospectif de l'historien, quand il projette sur un plan synchronique les différentes manières dont ils ont été reçus au cours de l'histoire. 
Avec le rejet de la polysémie, la théorie de l'expérience esthétique se retrouve à nouveau côte à côte avec la théorie déconstructiviste. Menke rappelle que Derrida veut distinguer la théorie de la polysémie de son idée de "dissémination». La plurivocité des signes se laisse toujours réintégrer dans une "téléologie herméneutique» 19 : tant que la polysémie des textes esthétiques se trouve rapportée à une "polythématique», elle reste prisonnière de «l'ordre du sens» 20 . Les diverses manières de comprendre un objet esthétique ont alors comme horizon un sens inépuisable qui est aussi l'expression de l'impossibilité d'exclure le concept d'un sens ultime. Cela étant dit, Menke ajoute que «la théorie de la polysémie est insuffisante [...] parce qu'elle ne se soucie pas de fonder la surabondance de sens qu'elle attribue à l'œuvre d'art. Elle ne répond pas à la question de savoir s'il s'agit d'un excès de sens, qui refuse d'être saisi dans un seul acte de compréhension [...], ou bien d'un excès propre aux signifiants esthétiques, qui se produit dans l'ajournement perpétuel de la compréhension"(Menke, p.85). Menke prétend que la négation de la compréhension propre à l'expérience esthétique et qui reste circonscrite dans cette expérience (contrairement au concept derridien de "dissémination") ne remet pas en cause le phénomène de la polysémie lui-même. En fait, la compréhension esthétique, qui est toujours ramenée à son point de départ sémiologique, à la matérialité brute, serait elle-même la condition de possibilité de la polysémie (Menke, p.86) - c'est une autre histoire.

b) La controverse entre l'esthétique de la négativité et l'herméneutique

Menke entend jouer de la controverse entre herméneutique et esthétique de la négativité pour dégager le contenu spécifique de l'expérience esthétique (Menke, pp.91-129). Alors que, selon Menke, l'herméneutique critique l'idée d'une culture esthétique autonome et voit en elle une «abstraction sémiologique qui escamoterait l'anticipation de sens inhérente à toute formation de signifiant»(Menke, p.92), il oppose la critique selon

19. Jacques Derrida, La dissémination, Paris : Seuil, 1972, p.371, (Menke. p.84).

20. Ibid., p.389 et p. 65, (Menke, p.84). 
laquelle la téléologie de l'herméneutique n'est pas en mesure d'envisager la compréhension depuis le stade de formation des signifiants, c'est-à-dire dans le champ de tensions qu'est la "lettre» tendu entre le sens et sa matérialité. La compréhension esthétique selon l'herméneutique est déjà effective parce qu'elle s'incrit, comme toute compréhension, dans un réseau de relations externes. Or pour Menke, comme on l'a dit, l'expérience esthétique doit être comprise comme un acte de compréhension qui, de manière immanente, est indéfiniment ajourné.

Menke mobilise Adorno dans la controverse entre l'esthétique de la négativité et l'herméneutique mais, inopinément, pas du tout en tant qu'auteur exemplaire. Adorno est plutôt vu comme le théoricien qui, tout en faisant émerger conceptuellement les catégories de la négativité esthétique, n'arrive pas à faire une théorie de la négativité esthétique autonome. En fait, la controverse entre l'herméneutique et l'esthétique de la négativité est immanente à l'œuvre même d'Adorno. L'esthétique dialectique et négative adornienne est un exemple de compénétration d'herméneutique et d'esthétique négative, dans la mesure où cette esthétique fonctionne elle-même dans des cercles herméneutiques ambivalents circonscrits dans la sphère de la formation des signifiants esthétiques, cercles ajournant l'avènement d'un sens plein mais ayant également une valeur allégorique pour la théorie sociale.

Les couples conceptuels de la Théorie esthétique associent des déterminations qui, prises isolément, désignent des aspects de l'objet esthétique : le tout, la partie, la forme, le matériau, la construction, la mimésis, etc. C'est seulement dans une réflexion ultérieure sur leur statut, et particulièrement sur les conditions de leur mise en œuvre, qu'elles se trouvent groupées par couples reliés les uns autres. Ce couplage implique que tout examen des objets esthétiques selon l'une des deux déterminations associées doit aussitôt être redoublé à partir de l'autre. Ainsi groupés, les concepts ne désignent pas immédiatement des aspects de l'objet, mais différentes perspectives dans lesquelles celui-ci s'offre à l'expérience esthétique. Le «tout" et le 
"détail», la "construction» et la "mimésis" représentent des aspects de l'explication, ou encore des dimensions de l'expérience des œuvres (Menke, p.94).

Mais, d'abord de tel binômes (tout/détail; construction/mimésis) restent "construits sur le mode paradoxal, [...] et expriment les mouvements contradictoires de la compréhension et de la subversion" (Menke, p.93).

L'idée menkienne d'une "hétéronomie latente» de la théorie esthétique d'Adorno renvoie au fait qu'en elle cœxistent une déconstruction du sens et une téléologie herméneutique. C'est d'ailleurs essentiellement cette hétéronomie que Menke voit retraduite dans la théorie d'A. Wellmer. L'expérience esthétique est devenue chez ce dernier un moyen permettant de découvrir des aspects habituellement cachés de la compréhension automatique (Menke, p.125) par le moyen d'une intensification du sens du quotidien qui fait perdre de vue la subversion du sens inhérente à l'expérience esthétique. Si la théorie adornienne est une herméneutique qui ne se sait pas, il reste que, selon Menke, on peut en dégager deux binômes conceptuels qui ont une portée systématique pour articuler la distinction entre la théorie de la négativité esthétique et l'herméneutique : le rapport entre tout et partie, qui eformule la relation entre les deux dimensions fondamentales de la compréhension d'un objet esthétique, entre le sens et le signifiant» - Menke, p.94 et sq. - (1); la relation entre le sens et sa formation, c'est-àdire entre «sens et mimésis» - Menke, p108 et sq. - (2).

1) Adorno écrit : "l'unité des œuvres d'art ne peut être ce qu'elle doit être, l'unité du divers : en synthétisant, elle porte atteinte au synthétisé, et ruine en lui la synthèse. Les œuvres souffrent autant du fait que leur totalité est médiatisée que de ce qui en elles est immédiat»21. Selon Menke, le paradoxe patent d'une telle formule s'éclaire dès lors que l'on voit la synthèse et le divers comme des niveaux d'énonciation différents dans la description des objets esthétiques. Alors que les deux niveaux d'énonciation s'opposent, les énoncés portant sur la synthèse (la totalité esthétique) reposent sur ceux concernant 
le divers (les détails esthétiques). De plus, la relation contradictoire de ces deux niveaux se répercute sur chacun des niveaux : par exemple, les énoncés sur les détails sont traités à la fois comme saisis dans la synthèse et opposés à elle (Menke, p.95). Pour leur part, "Les énoncés portant sur la totalité ne peuvent être réduits à la somme des énoncés portant sur les éléments" (Menke, p.97).

À partir de la compréhension de la synthèse et du divers dans l'œuvre d'art comme dimension d'interprétation paradoxale, l'auteur propose de ne plus comprendre le concept de "dissonance" esthétique, si central à la théorie d'Adorno, comme répondant d'un impératif de critique socio-historique. Le concept de "dissonance» serait plutôt inscrit d'abord dans la relation paradoxale du tout et des parties dans l'œuvre d'art que thématise le cercle herméneutique de la synthèse et du divers.

S'il est permis d'appeler "cercle herméneutique» le rapport paradoxal de ces niveaux d'énonciation interprétatifs, c'est précisément que leur relation n'est pas que paradoxe mais aussi dépendance réciproque. "L'esthétique de la négativité serait d'emblée vouée à l'échec si elle s'appuyait uniquement sur l'affirmation que ni le tout ni les parties de l'objet esthétique ne peuvent être exprimés. Car le fait que la réalité esthétique soit inexprimable ne justifie pas qu'on la conçoive comme soustraite à toute compréhension effective» (Menke, p.100). "Les aspects de l'œuvre qui, pris isolément, n'ont entre eux qu'un rapport paradoxal, se complètent dans la compréhension d'un contenu inexprimable» (Menke, p.101). Or, "que c'est [sic] une erreur d'assimiler inexprimabilité et incompréhensibilité des signes esthétiques, c'est ce qui ressort déjà d'un bref examen [...] du concept de cercle herméneutique» (Menke, p.100).

Cela dit, l'herméneutique philosophique, si elle explicite la théorie du cercle herméneutique - en reconnaissant à titre exemplaire son effectivité dans l'expérience esthétique - elle n'en marginalise pas moins la différence spécifique du mode de compréhension esthétique en le subordonnant à la visée de sens. On peut à juste titre se demander comment la relation de 
différents niveaux d'énonciation irréductiblement paradoxaux pourrait continuer d'en être une de dépendance réciproque si ce n'est à cause d'une visée de sens qui les dépasse tous deux? Menke répond de manière conséquente à cette question en distinguant d'abord la relation spécifique qui unit le sens et son support dans chaque mode de compréhension : «la compréhension historique détermine son objet comme document, la compréhension esthétique comme expression de son sens", alors que chez Gadamer, les deux concepts ne sont pas distingués (Menke, p.104)22. "La compréhension historique du sens d'un document dépasse l'objet donné, pour le rapporter à un contexte général, également donné à travers d'autres documents; la compréhension d'un sens esthétique s'enfonce au contraire dans son expression, pour le rapporter à sa manifestation singulière dans l'objet particulier» (Menke, pp.104-105).

Même si l'herméneutique admet avec sa théorie du «cercle herméneutique" la processualité négative de la formation du sens, cette processualité est aiguillée par une visée de sens extériorisé dans le partage dont il peut faire l'objet : «l'hésitation entre le matériau et la visée de sens s'achève dans l'œuvre comprise" (Menke, p.108). Pour sa part, l'esthétique de la négativité intègre l'attribution anticipée d'un sens dans le mouvement propre du processus négatif de formation des signifiants : la processualité de la formation des signifiants esthétiques se prolonge «jusque sur le plan de l'attribution du sens». (ibidem).

2) Évidemment, tout n'est pas dit sur l'apport de la dimension extra-esthétique au processus de formation du signifiant dans la compréhension esthétique telle que la conçoit l'esthétique de la négativité. D'après Menke, la dynamique d'un autre couple conceptuel de la théorie adornienne associant la mimésis à un antonyme variable - construction, forme, objectivation ou sens -, offre le complément théorique que l'on cherche. C'est dans ce couple conceptuel que s'affirmerait la condition rendant essentielle la différence entre la façon de concevoir le rapport des signifiants et de la signification dans l'esthétique de la négativité et dans l'herméneutique.

22. Cf. H.-G. Gadamer, Vérité et méthode, op. cit., p.179. 
Menke cite Adorno : “L'analyse n'approche l'œuvre d'art que lorsqu'elle saisit de façon processuelle le rapport des composantes entre elles, et non lorsqu'elle la réduit par décomposition à de prétendus éléments premiers ${ }^{23}$ ". Ceci suppose, selon Menke, que la processualité de l'expérience esthétique est «à la fois acte de compréhension et (ré-) effectuation mimétique» (Menke, p.111). D'après l'auteur, "le sens esthétique est un sens compris - ce qui signifie qu'il se rapporte sur le mode de la répétition à un sens connu ailleurs"(ibidem). II va donc s'attarder à la spécification de la différence entre le concept de reconnaissance dans l'herméneutique et l'esthétique de la négativité.

L'auteur souligne qu'«en affirmant que l'esthétique se résout dans l'herméneutique, Gadamer suggère un parallèle entre l'étrangeté des signes esthétiques et celle des signes provenant d'une époque révolue» (Menke, p.113). C'est pour se donner une "logique" de l'interprétation visant une compréhension intégrante de ce qui semble incompréhensible que l'herméneutique prétend que toute compréhension implique la structure du préjugé. Un préjugé n'est pas une idée fausse, mais le reflet d'un enracinement contextuel, qui fonde la compréhension de l'altérité : «les préjugés sont librement repris, mais nullement créés ou fondés dans leur validité par un acte libre de l'intelligence» 24 . Chaque situation d'interprétation constitue par ses préjugés un horizon, et l'étrangeté des signes d'époques révolues n'empêche pas le fait qu'on rencontre ces signes comme les indices d'un horizon historique que l'on comprend comme structure de préjugé. Selon l'herméneutique, nous dit Menke, "comprendre suppose que notre faculté de compréhension ne soit que partiellement contrariée par l'étrangeté du signe» (Menke, p.115), ce qui n'est pas le cas dans l'expérience esthétique, bien au contraire. Cela devient patent si l'on décide de thématiser l'expérience esthétique comme une "fusion d'horizons". Si l'on comprend cette fusion comme celle de l'horizon créé par la matérialité du signe esthétique et celui

23. T. W. Adorno, Théorie esthétique, op. cit., p.226, (Menke, p.110).

24. H.-G. Gadamer, op. cit., p.119, (Menke, p.113). 
du spectateur avec son expérience propre, la perspective se renverse : ce n'est plus l'individu et son expérience qui posent le regard sur une réalité relativement étrangère, mais la radicale altérité immanente au lien paradoxal entre la matérialité du signe et le sens anticipé qui pose le regard sur cet individu. «La compréhension esthétique révèle la zone obscure de notre propre horizon" (Menke, p.116). C'est de cette façon bien spécifique que la compréhension esthétique est reconnaissance : elle "est une répétition (non représentationnelle) de l'horizon propre du sujet, horizon qui existe toujours déjà en dehors de sa représentation esthétique, tout en n'étant pas encore connu» (Menke, p.117).

L'auteur insiste aussi sur le fait que la mimésis adornienne renvoie à un mode de connaissance par identification aconceptuelle. II rappelle Adorno : "Ce qui est essentiellement mimétique attend une attitude mimétique. Si les œuvres d'art n'imitent rien d'autre qu'elles-mêmes, alors seul les comprend celui qui les imite 25 ", c'est-à-dire celui qui les recrée «objectivement de l'intérieur en en faisant l'expérience ${ }^{26}$ ». Cela prend un sens particulier pour Adorno dans le cadre de l'expérience artistique moderne et assumée - particulièrement dans la musique 27 - où plus aucune règle a priori n'est opérante. «L'expérience esthétique est mimétique, parce qu'en l'effectuant nous reproduisons de l'intérieur, dans le jeu de ses forces, le processus par lequel les dimensions de la compréhension esthétique se trouvent rapportées l'une à l'autre" écrit Menke (Menke, p. 121).

Alors que Gadamer pense que la tension entre l'orientation de la parole vers un sens et l'autoprésentation de son apparaître dans la reprise mimétique se résout dans un jeu de renvois réciproques, selon Menke, la théorie de la négativité refuse de trancher la question parce que le véritable contenu de l'expérience esthétique est cette tension même. "L'herméneutique soumet le processus de l'expérience esthétique à la téléologie

25. T. W. Adorno, Théorie esthétique, op. cit., p.166, (Menke, p.118).

26. Ibid., p.160, (Menke, p.119).

27. Cf. notamment, T.W Adorno, Quasi una fantasia, Paris : Gallimard, 1982, p.289340. 
d'une compréhension effective, parce qu'elle a d'avance décrété que l'expérience esthétique constitue le médium dans lequel une signification intensifiée s'offre à la compréhension" (Menke, p.123), en l'occurrence, "dans le poème, selon Gadamer, la venue au langage est comme une insertion dans les rapports ordonnés par quoi la "vérité" de ce qui est dit est étayée et garantie ${ }^{28}$ ". D'une manière générale, les théories herméneutiques "subordonnent la ré-effectuation immanente et seule réellement "esthétique" de la compréhension au postulat selon lequel l'expérience esthétique répète, en les transformant, des actes d'appréhension du sens accomplis en dehors de la sphère de l'art" (ibidem). En cela, selon Menke, les herméneutiques ne font que subordonner l'expérience esthétique à des actes de compréhension extra-esthétiques et nous empêchent d'en comprendre le potentiel subversif à l'égard d'actes de compréhension stabilisés, automatisés dans les champs extraesthétiques.

En bout de ligne, Menke met l'esthétique de la négativité et l'herméneutique dans un rapport antagoniste irréductible : alors que résoudre la tension immanente à la reprise mimétique est pour l'herméneutique un moyen d'intensifier la signification esthétique, pour l'esthétique de la négativité, maintenir cette tension c'est assumer souverainement le potentiel subversif de l'expérience esthétique, la part d'altérité inhérente au sens.

\section{III- Remarques critiques}

Même si l'on peut être d'accord avec l'ensemble des critiques que Menke formule à l'égard de l'herméneutique philosophique dans son rapport à l'art, ne nous est-il pas permis de penser le dépassement de cette herméneutique autrement que sous la forme du repli sur une esthétique de la négativité? Si l'herméneutique conçoit que «la reprise mimétique de la genèse du sens dans son irréductible dimension énergétique et dynamique acquiert elle-même une fonction sémiotique» (Menke, p.122), n'y a-t-il pas moyen d'imaginer cette fonction autrement qu'à la manière de l'herméneutique qui 
jusqu'à maintenant en fait une «intensification» du sens? Un tel dépassement ne nous permettrait-il pas de concevoir la dimension herméneutique de la théorie esthétique adornienne autrement que comme l'agent d'une "hétéronomie latente", attendu qu'elle impliquerait des actes de compréhension liés à des considérations extra-esthétiques? Car il ne faut pas oublier que l'auteur a lui-même montré comment le rapport des concepts de tout et de partie dans l'esthétique adornienne était constitutivement celui du cercle herméneutique. N'est-ce pas d'une certaine façon aussi dire que la théorie adornienne est, de manière patente et irréductible, une herméneutique, même après avoir été amputée de ses prétentions socio-critiques? II me semble que Menke ouvre un espace pour cette interrogation dans la mesure où il a lui-même énoncé une thèse différenciant les cercles herméneutiques : "Le cercle herméneutique relie, dans la compréhension historique le sens et son contexte, dans la compréhension esthétique, le sens et son expression" (Menke, p.105). Pourquoi faire cette différenciation et la laisser tomber en faveur d'une critique définitive de l'herméneutique?

Je propose plutôt de réinterpréter la théorie d'Adorno comme une herméneutique de la dialectique du sens et de l'expression qui ne renvoie plus à une intensification du sens, mais au contraire à des médiations symboliques - les œuvres d'art - où l'ambiguïté des signifiants est constitutive d'un intérêt expérienciel pour la communication de la frange du communicable ${ }^{29}$. La processualité mimétique de l'expérience artistique ne serait plus vue comme le chemin d'une intensification, non plus que comme celui d'une subversion du sens, mais comme l'incarnation même de la fragilité communicationnelle immanente de l'art. Tout cela implique des présupposés anthropologiques sur l'expression de soi et la communication que je ne peux aborder ici.

Cela étant dit, il nous est au moins permis de voir la thèse de l'herméneutique philosophique d'une intensification du sens dans l'expérience esthétique comme une réponse à ce qu'elle

29. Voir Michel Ratté, "Autonomie de l'art et communication...", op. cit., p.373386. 
voit se mettre en œuvre dans l'autonomisation de l'art. L'idée d'une intensification du sens constitutive de l'expérience esthétique est une réponse philosophique au fait que pour l'herméneutique, l'art, en tant que sphère autonomisée, risque de ne plus avoir de sens. Or, on l'a dit, on pourrait assumer qu'il est en soi fragile par la fin qui met en jeu ses médiations subtiles : communiquer la frange du communicable. Cette fragilité n'est pas en soi l'indice de sa perte de fonction symbolique. Pourtant, par cette fragilité, l'art prête le flanc à une surdétermination compensatoire de son sens, surdétermination induite par les contraintes de la circulation sociale de l'art à titre de marchandise ${ }^{30}$.

Dans cette condition particulièrement exacerbée aujourd'hui, on peut se demander d'où la subversion souveraine, voire "contraignante» que Menke voit mise en œuvre dans l'expérience esthétique moderne peut tirer son intensité propre - car si l'herméneutique parle d'intensification du sens, comment comprendre la subversion souveraine du sens autrement que comme une intensité du non-sens? La question me semble scrupuleusement esquivée chez lui dans le détour d'une discussion toute formelle du rapport entre l'expérience esthétique et la raison.

On l'a vu, Menke réattribue à la souveraineté esthétique un lieu plutôt qu'une liberté sur la base du fait que l'autonomie de sa rationalité, qui consiste en une critique de la raison, rencon-

30. Ibid., p. 386-390. Le fait que dans ce texte, je reformule le problème communicationnel de l'art en termes de fragilité communicationnelle vise aussi à opérer un recentrement de la question fondamentale chez Habermas du rapport entre la vie quotidienne et l'institution artistique. En liant une réception exotérique de l'art à sa liberté ésotérique, Habermas prétend formuler la condition d'une fluidification communicationnelle en soi légitime de l'institution artistique, qui ne doit pas se contenter de vivre comme une fatalité sa réinterprétation à grande échelle. Cela dit, compte tenu de la fragilité communicationnelle de l'art et de son investissement par les discours qui contraignent sa réception dans le marché, c'est, il me semble, à l'égard de la culture populaire non pas en tant que récit d'une réception alternative du "grand art" mais dans la culture populaire en tant qu'elle se commet dans la production d'œuvre d'art que l'on doit voir le vrai tonique de la fluidification de l'institution artistique. Pour sa part, Menke a déjà sacrifié ce projet habermassien en le considérant sans plus comme une trahison de l'autonomie de l'art (Cf. Menke, p.289-290). 
tre tout de même sa limite dans le respect des autres rationalités. La souveraineté de la négativité esthétique traduit la possibilité d'amener les contenus d'expériences réalisés dans d'autres sphères de rationalité au sein de sa sphère propre. Mais en admettant comme Menke que cette souveraineté se distingue de celle de la déconstruction en ce qu'il lui incombe en quelque sorte de réattribuer sa négativité aux autres sphères de rationalité à titre de bénéfice a posteriori pour ces sphères, n'est-on pas en train de montrer que la négativité n'est pas foncièrement souveraine? De toute évidence, la négativité esthétique reste logiquement dépendante de la raison d'ailleurs, Menke ne le contesterait probablement pas. Mais par le fait même, la proposition menkienne selon laquelle cette souveraineté esthétique produit une condition "postesthétique» des autres discours revient à dire que l'expérience esthétique a une fonction heuristique qui demande plus qu'une description en termes de postériorité logique. La théorie de Menke ne peut pas se satisfaire de cette formalité qui avait d'abord servi à faire une distinction entre la déconstruction et l'esthétique négative. En d'autres mots, si la négativité esthétique a le pouvoir "contraignant» de nous faire "acquérir une nouvelle image des autres modes de discours" (Menke, p.197) - ce qui revient à affirmer sa fonction thérapeutique pour la raison - il faudrait en expliciter plus avant les effets historiques pour convaincre, surtout après que l'auteur ait révoqué les présupposés anthropologiques de l'herméneutique et de la théorie adornienne, et qu'il ait simplement greffé à sa théorie formelle une reformulation «négativiste» de la théorie de la beauté.

Michel Ratté 